\title{
LncRNA TRG-AS1 stimulates hepatocellular carcinoma progression by sponging miR-4500 to modulate BACH1
}

\author{
Xuehu Sun ${ }^{1}$, Yeben Qian ${ }^{2 *} \mathbb{D}$, Xingyu Wang ${ }^{1}$, Rongge $\mathrm{CaO}^{3}$, Jianlin Zhang ${ }^{1}$, Weidong Chen ${ }^{1}$ and Maoyong Fang ${ }^{1}$
}

\begin{abstract}
Background: T cell receptor gamma locus antisense RNA 1 (TRG-AS1) has been reported to involve in the progression of glioblastoma, however the role and its underlying molecular mechanism in hepatocellular carcinoma (HCC) remain unknown.

Methods: Quantitative real-time polymerase chain reaction (RT-qPCR) was applied to detect TRG-AS1 expression in HCC cells. Besides, the proliferation abilities of HCC cells were assessed by colony formation and EdU assays. The migratory and invasive abilities of HCC cells were examined by transwell assays. Imunofluorescence staining (IF) was used to analyze the epithelial-mesenchymal transitions (EMT). The interaction among TRG-AS1, miR-4500 and BTB domain and CNC homolog 1 (BACH1) were proofed by means of RIP and RNA pull down and luciferase reporter assays.
\end{abstract}

Results: TRG-AS1 was conspicuously overexpressed in HCC cells. TRG-AS1 silencing apparently suppressed HCC cell proliferation, migration, invasion and epithelial-mesenchymal transition (EMT). Mechanism exploration revealed that TRG-AS1 acted as a molecular sponge of miR-4500 to regulate BACH1. MiR-4500 silencing or BACH1 overexpression in BACH1-downregulated cells fully rescued cell proliferation migration, invasion and EMT progress.

Conclusion: TRG-AS1 regulates HCC progression by targeting miR-4500/BACH1 axis.

Keywords: TRG-AS1, miR-4500, BACH1, HCC

\section{Background}

Hepatocellular carcinoma (HCC) is a type of primary liver cancer, which is regarded as the fifth common cancer worldwide [1]. There are many risk factors to implicate in this course such as chronic infection and cirrhosis by hepatitis B virus (HBV) and cirrhosis. [2]. With the development of medical technology and improvement of health system, more and more advanced therapeutic managements have been applied to treat HCC patients, such as liver transplantation [3], proton beam therapy

\footnotetext{
*Correspondence: qianyeben@hotmail.com

2 Department of Hepatobiliary Surgery, the First Affiliated Hospital of Anhui Medical University, No.218 Jixi Road, Hefei 230000, Anhui, China

Full list of author information is available at the end of the article
}

[4], percutaneous/laparoscopy-assisted radiofrequency ablation [5] and etc. Nevertheless, the prognosis of HCC patients remains unfavorable. Therefore, exploration of mechanism associated with the initiation and progression of $\mathrm{HCC}$ is essential for providing a new insight for the treatment of HCC.

Noncoding RNAs (ncRNAs) are consisted of short and long ncRNAs, which are related with cancers [6]. Recent years, the underlying molecular mechanism of lncRNAs have been discussed in multiple cancers, including HCC $[7,8]$. For instance, PVT1 silencing significantly suppresses prostate cancer cell growth [9]. HANR promotes tumorigenesis of $\mathrm{HCC}$ and enhances chemoresistance [10]. In addition, RGMB-AS1 and CDKN2B-AS1 accelerate tumor metastasis of HCC $[11,12]$.

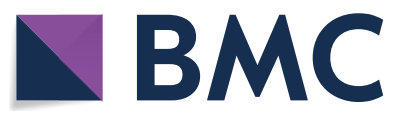

(c) The Author(s) 2020. This article is licensed under a Creative Commons Attribution 4.0 International License, which permits use, sharing, adaptation, distribution and reproduction in any medium or format, as long as you give appropriate credit to the original author(s) and the source, provide a link to the Creative Commons licence, and indicate if changes were made. The images or other third party material in this article are included in the article's Creative Commons licence, unless indicated otherwise in a credit line to the material. If material is not included in the article's Creative Commons licence and your intended use is not permitted by statutory regulation or exceeds the permitted use, you will need to obtain permission directly from the copyright holder. To view a copy of this licence, visit http://creativeco mmons.org/licenses/by/4.0/. The Creative Commons Public Domain Dedication waiver (http://creativecommons.org/publicdomain/ zero/1.0/) applies to the data made available in this article, unless otherwise stated in a credit line to the data. 
LncRNAs can exert tumor-suppressing or tumor-promoting functions by sponging miRNAs to up-regulate downstream mRNAs [13]. For example, MCM3AP-AS1 plays an oncogenic role in HCC by absorbing miR-194-5p to promote FOXA1 expression in HCC [14]. T cell receptor gamma locus antisense RNA 1 (TRG-AS1) has been determined to be oncogenic in glioblastoma [15]. However, whether TRG-AS1 had the effect on HCC cellular processes remains to be unveiled.

In this study, we focused on the role and underlying mechanism of TRG-AS1 in HCC.

\section{Materials and methods \\ Cell lines}

Human liver immortalized cell line THLE-2 and human HCC cell line SK-HEP-1 were both procured from ATCC (Manassas, VA, USA). Human HCC cell line HCCLM3 was procured from China Center for Type Culture Collection (CCTCC; Wuhan, China), MHCC97-L cell line was procured from Procell Life Science \& Technology Co., Ltd. (Wuhan, China). MHCC97-H and Huh-7 cell lines were purchased from the Cell Bank of the Chinese Academy of Sciences (Shanghai, China). THLE-2 cell was cultured in BEGM (Lonza/Clonetics Corporation, Walkersville, MD, USA). HCCLM3, Huh-7 and SK-HEP-1 was cultured in DMEM (Gibco, Grand Island, NY, USA) with 10\% fetal bovine serum (FBS; Gibco), plus 1\% penicillin/ streptomycin (Thermo Fisher Scientific, Grand Island, NY, USA). MHCC97-H and MHCC97-L cells were cultured in RPMI-1640 with $10 \%$ FBS. All cells were maintained in a $5 \% \mathrm{CO}_{2}$ incubator at $37^{\circ} \mathrm{C}$.

\section{Total RNA extraction and RT-qPCR}

Total RNA was extracted with application of TRIzol Reagent (Invitrogen, Carlsbad CA, USA), and then was processed with PrimeScript Reverse Transcriptase Kit (Takara, Shiga, Japan). The acquired cDNA template was used for quantitative analysis with SYBR Green PCR Kit (Takara) on ABI Prism 7900HT sequence detector (Applied Biosystems, Foster City, CA, USA). At length, results were calculated by $2^{-\Delta \Delta C t}$ method by using GAPDH or U6 as internal control.

\section{Cell transfection}

The shRNAs specifically targeting TRG-AS1 and BACH1 were designed and constructed by GenePharma (Shanghai, China), together with the negative control (NC)shRNAs. The full-length of BACH1 cDNA sequence was cloned into pcDNA3.1 vector (Invitrogen) for overexpression. The empty pcDNA3.1 vector was employed in $\mathrm{NC}$ group. In addition, miR-4500 inhibitor and miR4500 mimics were synthesized by Ribobio (Guangzhou, China), along with the NCs. All these were transfected into HCCLM3 and Huh-7 cells for $48 \mathrm{~h}$ with application of Lipofectamine 3000 (Invitrogen). This assay contained three independent repeats.

\section{Colony formation assay}

After transfection, HCCLM3 and Huh-7 cells were planted into 6-well plates at a density of 800 cells per well and incubated for 14 days. Then, cells were fixed by methanol for staining in $0.5 \%$ crystal violet solution (SigmaAldrich). The number of colonies was counted manually. This assay contained three independent repeats.

\section{EdU assay}

transfected HCCLM3 and Huh-7 cells were collected and planted into 96-well plates $\left(1 \times 10^{4}\right.$ cells per well), followed by addition of EdU assay kit (Ribobio) for $2 \mathrm{~h}$ at $37{ }^{\circ} \mathrm{C}$. After that, cells were treated in DAPI solution for $5 \mathrm{~min}$ at room temperature. At length, fluorescence microscope (Olympus, Tokyo, Japan) was used for detecting proliferative cells. This assay contained three independent repeats.

\section{Transwell assay}

$2 \times 10^{4}$ cells in serum-free medium were prepared and added into the upper chamber of Transwell inserts (24well; Corning Incorporated, Corning, NY, USA) for cell migration assay. As for cell invasion assay, the chamber was coated with Matrigel membrane (BD Biosciences, Franklin Lakes, NJ, USA). Lower chamber was filled with $100 \%$ complete medium. Migrated and invaded cells were visualized by crystal violet staining after fixing by $4 \%$ PFA. 5 random fields were chosen and observed by optical microscope (Olympus). This assay contained three independent repeats.

\section{Immunofluorescence staining (IF)}

Cells on the culture slides were collected after adhered to the slides, and then washed in PBS three times. After that, cells were fixed for $10 \mathrm{~min}$ and blocked in 5\% BSA for $10 \mathrm{~min}$, followed by culture with primary antibodies against E-cadherin and N-cadherin (Cell Signaling Technology, St Louis, Missouri, USA) at $4{ }^{\circ} \mathrm{C}$ overnight. Afterwards, the secondary antibody was added before DAPI staining. The stained cells were visualized using fluorescence microscope (Olympus). This assay contained three independent repeats.

\section{Fluorescent in situ hybridization (FISH)}

The FISH probe specifically targeting TRG-AS1 was procured from Ribobio and used as guided. Cells were cultivated with the probe in the hybridization buffer, and then stained in Hoechst solution, analyzed by fluorescence 
microscope (Olympus). This assay contained three independent repeats.

\section{Nucleus-cytoplasm fractionation}

The subcellular location of TRG-AS1 was also analyzed via nucleus-cytoplasm fractionation assay with application of Cytoplasmic \& Nuclear RNA Purification Kit (Norgen, Belmont, CA, USA). Cell cytoplasm and cell nucleus were separated by cell fractionation buffer. After centrifugation, the RT-qPCR was conducted for the content of TRG-AS1, U6 and GAPDH were used as internal controls. This assay contained three independent repeats.

\section{RNA immunoprecipitation (RIP)}

RIP assay was studied by use of control IgG and human Ago2 antibodies in RIP buffer. The cell lysates were prepared using RIP lysis buffer, and then cultivated with the antibodies-bound magnetic beads for $6 \mathrm{~h}$. After that, the immunoprecipitates were collected for RT-qPCR analysis. This assay contained three independent repeats.

\section{RNA pull down}

RNA pull down analysis was carried out with application of the Pierce Magnetic RNA-Protein Pull-Down Kit (Thermo Fisher Scientific, Waltham, MA, USA). Cell proteins were mixed with magnetic beads and the biotinlabeled probes for TRG-AS1 or miR-4500. After collecting the RNA-protein mixture, RT-qPCR was used for analysis. This assay contained three independent repeats.

\section{Luciferase reporter assay}

The fragments of TRG-AS1 or BACH1 3'UTR, which contained the wild-type and mutated binding sites of miR-4500, were first prepared for inserting into the pmirGLO dual-luciferase vectors (Promega, Madison, WI, USA). After that, the acquired constructs were co-transfected into HCCLM3 and Huh-7 cells with miR-4500 mimics or NC mimics. Forty-eight hours later, the relative luciferase activity was examined by Dual-luciferase reporter assay system (Promega). This assay contained three independent repeats.

\section{Western blot}

Total protein was extracted from HCC cells using RIPA lysis buffer. Extracted proteins were subsequently loaded on SDS-polyacrylamide gels and then transferred to PVDF membranes which were blocked in 5\% non-fat milk for $1 \mathrm{~h}$ and then incubated with primary antibodies against E-cadherin (1:1000, ab238099, Abcam, USA), N-cadherin (1:5000, Abcam) and GAPDH (1:2000, Abcam) at $4{ }^{\circ} \mathrm{C}$ overnight. Then, the membranes were incubated with anti-rabbit secondary antibodies (1:5000;
Abcam). Finally, the signals were detected on the ECL chemiluminescence system.

\section{Statistical analyses}

Each assay of this study contained three independent repeats, and results were shown as the mean \pm SD. The group difference was analyzed by one-way ANOVA or Student's $t$ test, with application of GraphPad PRISM 6 (GraphPad, San Diego, CA, USA). The experimental data were collected when $\mathrm{p}<0.05$.

\section{Results \\ TRG-AS1 plays a tumor-promoting role in HCC}

To thoroughly investigate the role of lncRNA TRG-AS1 in HCC, we initially conducted RT-qPCR to evaluate its expression in HCC cells (HCCLM3, MHCC97-H, Huh-7, MHCC97-L and SK-HEP-1) and normal cell THLE-2. Compared with the THLE-2 cell, TRG-AS1 was obviously up-regulated in HCC cells, especially in HCCLM3 and Huh-7 cells (Fig. 1a). Then, we wondered TRG-AS1 whether affected the biological behaviors of HCC cells. RT-qPCR detected that TRG-AS1 expression was effectively cut down in the HCCLM3 and Huh-7 cells transfected with sh-TRG-AS1\#1 and sh-TRGAS1\#2 compared to sh-NC group (Fig. 1b). Subsequently, we observed that TRG-AS1 knockdown effectively restrained the number of colonies and EdU-positive cells (Fig. 1c, d), which suggested that TRG-AS1 knockdown prevented the abilities of HCC cells to proliferate. Similar effects were also observed in cell invasion and migration (Fig. 1e). Furthermore, both mRNA and protein levels of $\mathrm{E}$-cadherin and $\mathrm{N}$-cadherin were detected in TRG-AS1-silenced HCC cells. Both levels of E-cadherin were enhanced after silencing of TRG-AS1, whereas that of N-cadherin were impeded by TRG-AS1 knockdown (Additional file 1: Figure S1A, B). The same tendency was observed through imunofluorescence staining analysis (Fig. 1f). Collectively, TRG-AS1 exerts a tumor-promoting role in HCC.

\section{TRG-AS1 binds to miR-4500 in HCC cells}

The potential mechanism associated with TRG-AS1 in HCC was investigated. Subcellular fractionation assay primarily uncovered that lncRNA TRG-AS1 was mostly distributed in the cytoplasm of HCC cells and the same result was obtained in FISH assay (Fig. 2a, b). In addition, TRG-AS1 was visibly abundant in Anti-Ago2 complex compared to Anti-IgG complex, which was confirmed by RIP assay (Fig. 2c). Based on these results, we subsequently explored the downstream targets of TRG-AS1. Firstly, we applied online databases ENCORI (http://starbase.sysu.edu.cn/) and miRDB (http://mirdb .org/) to screen out 20 miRNAs that could interact with 


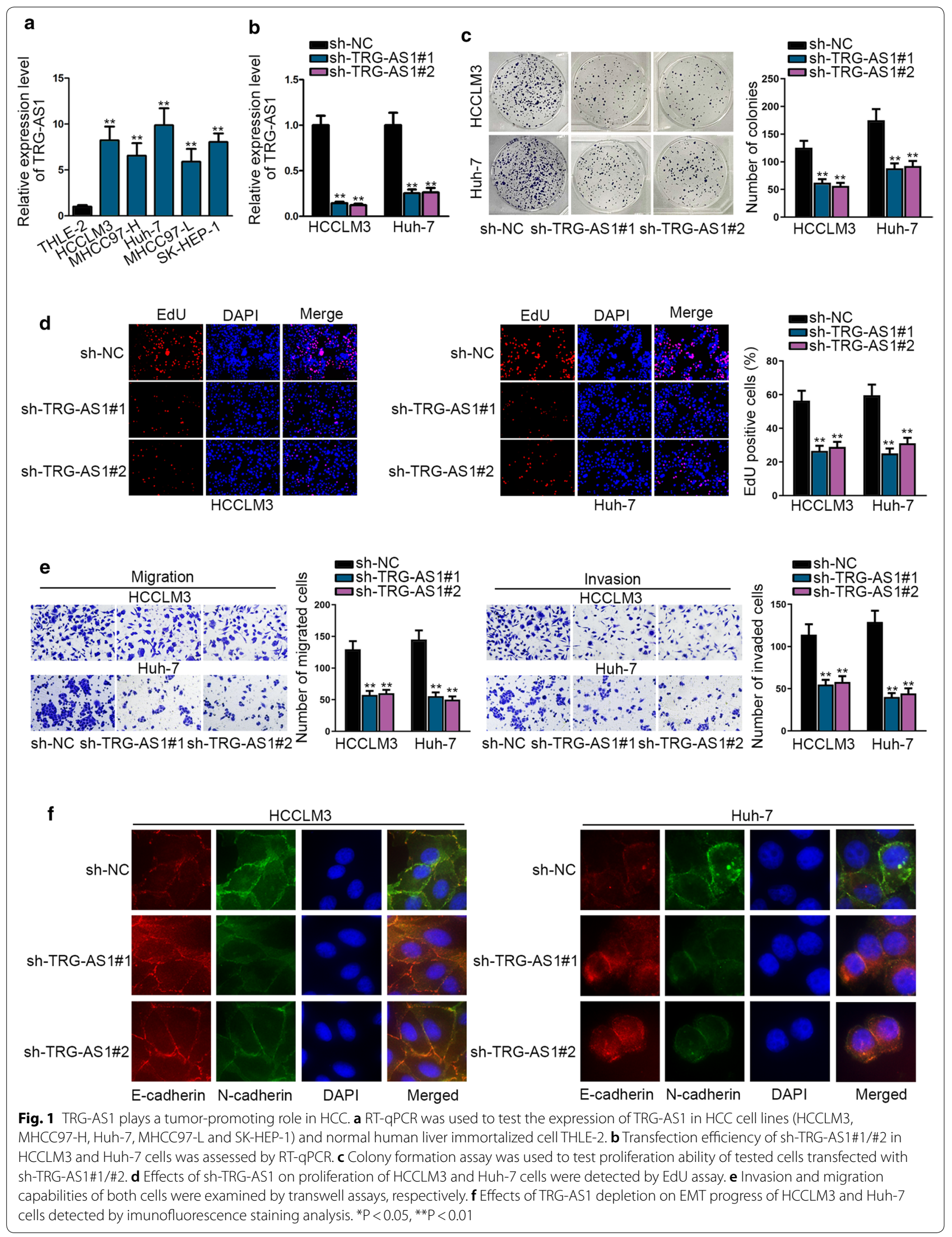




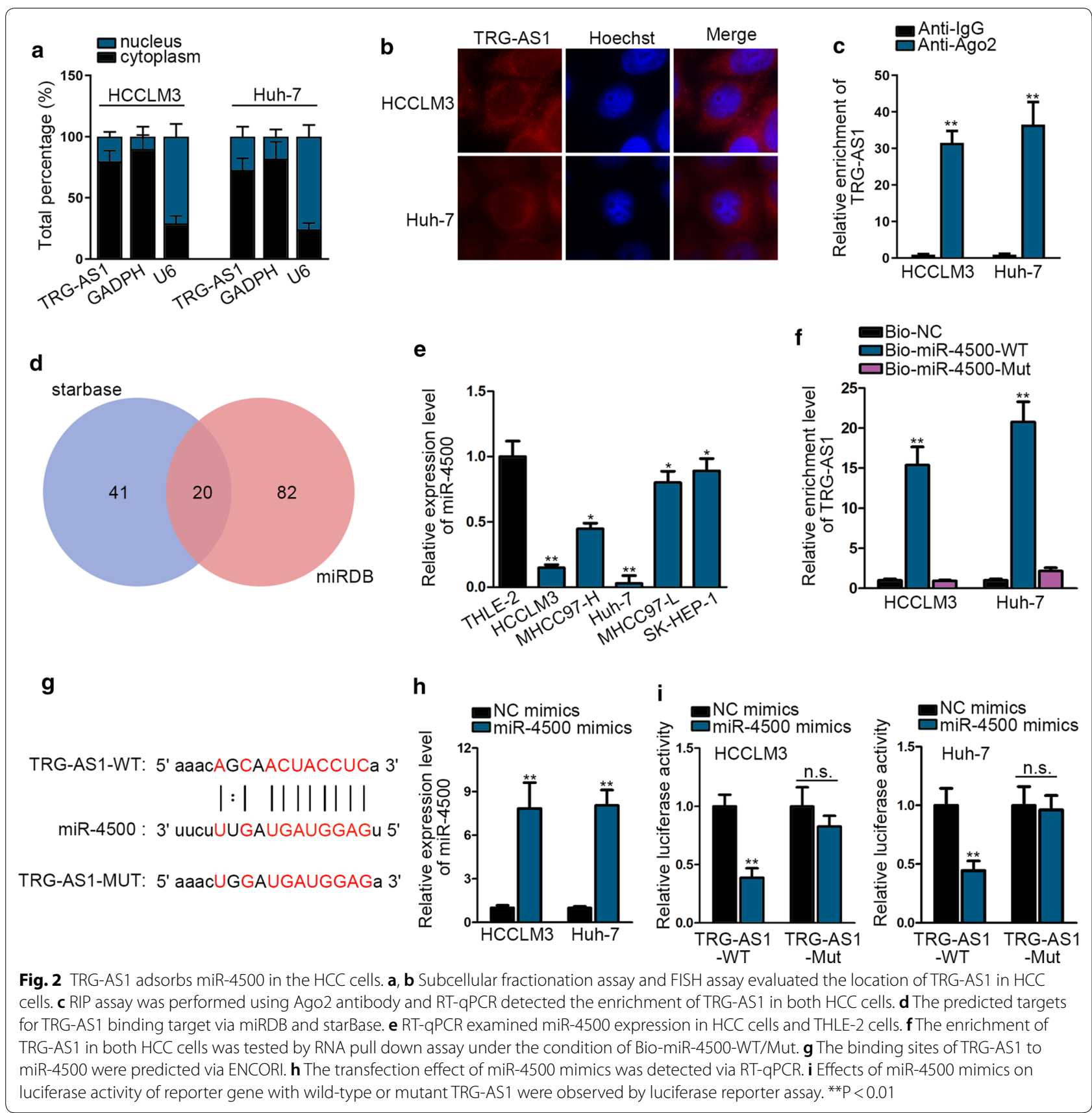

TRG-AS1 (Fig. 2d). RNA pull down assay subsequently revealed that miR-4500 was apparently enriched in TRG-AS1 biotin probe group compared with NC biotin probe (Additional file 2: Figure S2A). As presented in Fig. 2e, miR-4500 expression was down-regulated in HCC cells. RNA pull down assay was used to further determine the relevance between TRG-AS1 and miR4500. The results showed that the enrichment of TRGAS1 was increased by Bio-miR-4500-WT instead of Bio-miR-4500-Mut by comparing with Bio-NC group
(Fig. 2f). In addition, ENCORI also exhibited the binding sites between miR-4500 and TRG-AS1 (Fig. 2g). What's more, miR-4500 expression was enhanced in the HCCLM3 and Huh-7 cells transfected with miR4500 mimics (Fig. 2h). Finally, luciferase reporter assay clearly indicated that the decreased luciferase activity of TRG-AS1 wild type was induced by miR-4500 mimics by contrast with NC-mimics group (Fig. 2i). Taken together, all data explained that TRG-AS1 adsorbs miR4500 in HCC cells. 
MiR-4500 suppresses HCC cells proliferation, invasion, migration and EMT

According to above data, miR-4500 was distinctly downregulated in HCC cells. Then, we tried to explore the function of miR-4500 in HCC. As disclosed in colony formation assay and EdU assay, the number of colonies and EdU-positive cells exhibited the decreased tendency (Fig. 3a, b). Besides, the number of migrated and invaded cells were suppressed by miR-4500 mimics (Fig. 3c). Furthermore, the increased level of E-cadherin and the reduced level of $\mathrm{N}$-cadherin were observed in cells with ectopic expression of miR-4500 (Additional file 3: Figure S3A, B). Meanwhile, the results of imunofluorescence staining analysis unveiled that miR-4500 overexpression reversed EMT progress by increasing E-cadherin expression and declining N-cadherin expression (Fig. 3d). Collectively, miR-4500 suppresses HCC cell proliferation and invasion and migration as well as EMT progress.

\section{BACH1 is modulated by the TRG-AS1 and miR- 4500}

Subsequently, we probed the possible targets of miR-4500 in HCC. There were five mRNAs selected out via RNA22, PicTar, TargetScan and miRmap datasets (Fig. 4a). And then RT-qPCR analysis manifested that BACH1 expression was observably decreased in HCCLM3 and Huh-7 cells treated with miR-4500 mimics, while others had no obvious changes (Additional file 4: Figure S4A). Moreover, $\mathrm{BACH} 1$ expression was also down-regulated due to
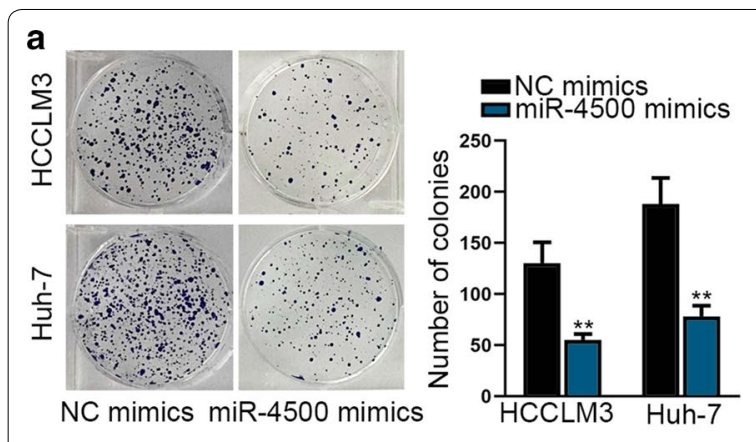

b

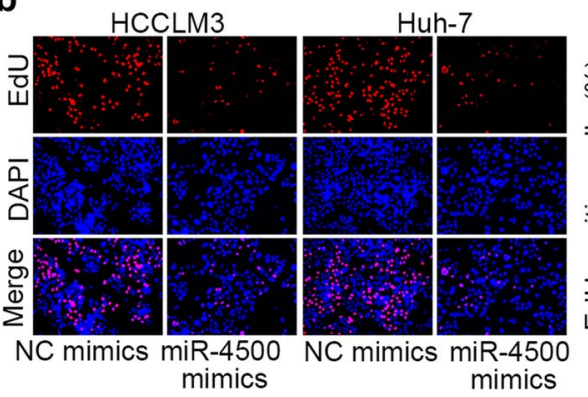

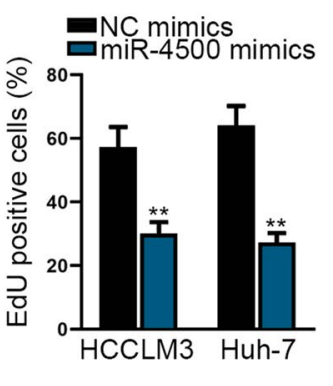
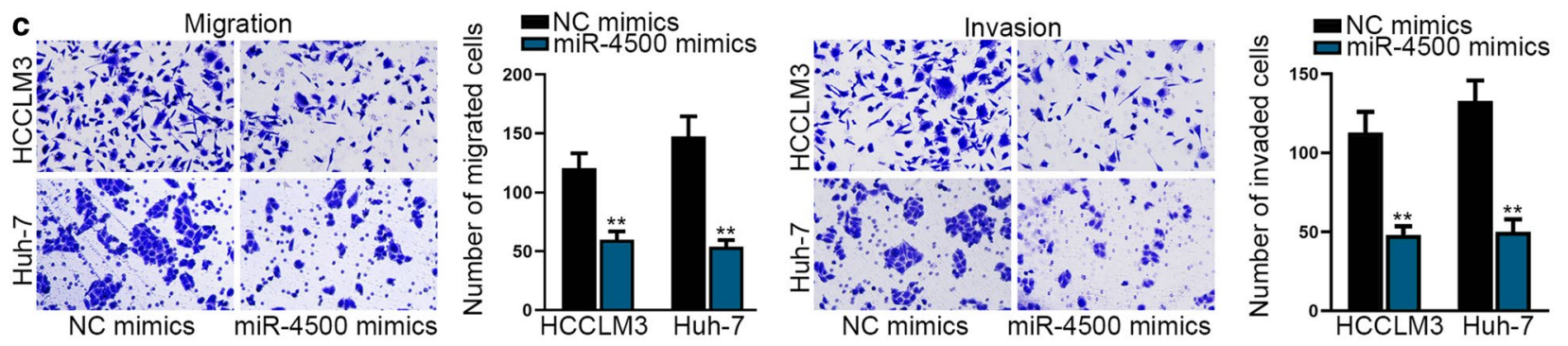

d

HCCLM3
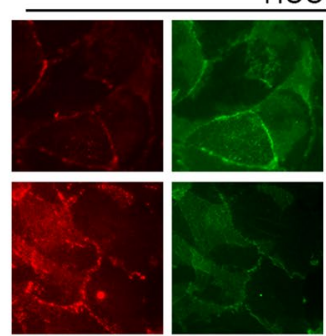

E-cadherin N-cadherin

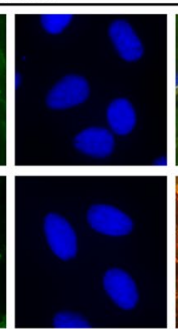

DAPI

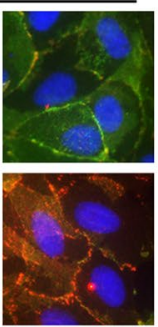

Merged

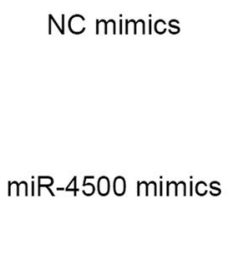

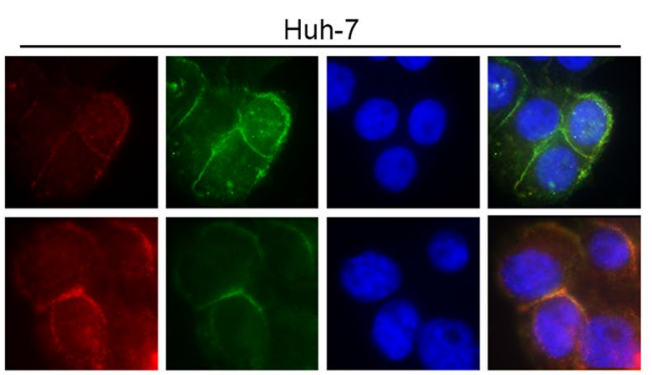

E-cadherin $\mathrm{N}$-cadherin
DAPI evaluate colony and proliferation capacities of HCCLM3 and Huh-7 cells in the context of miR-4500 mimics. c The transwell assay evaluated the invasion and migration abilities of HCCLM3 and Huh-7 cells after miR-4500 was up-regulated. $\mathbf{d}$ Under the circumstance of miR-4500 overexpression, EMT progress in HCC was measured with the assistance of imunofluorescence staining analysis. ${ }^{* * P}<0.01$ 

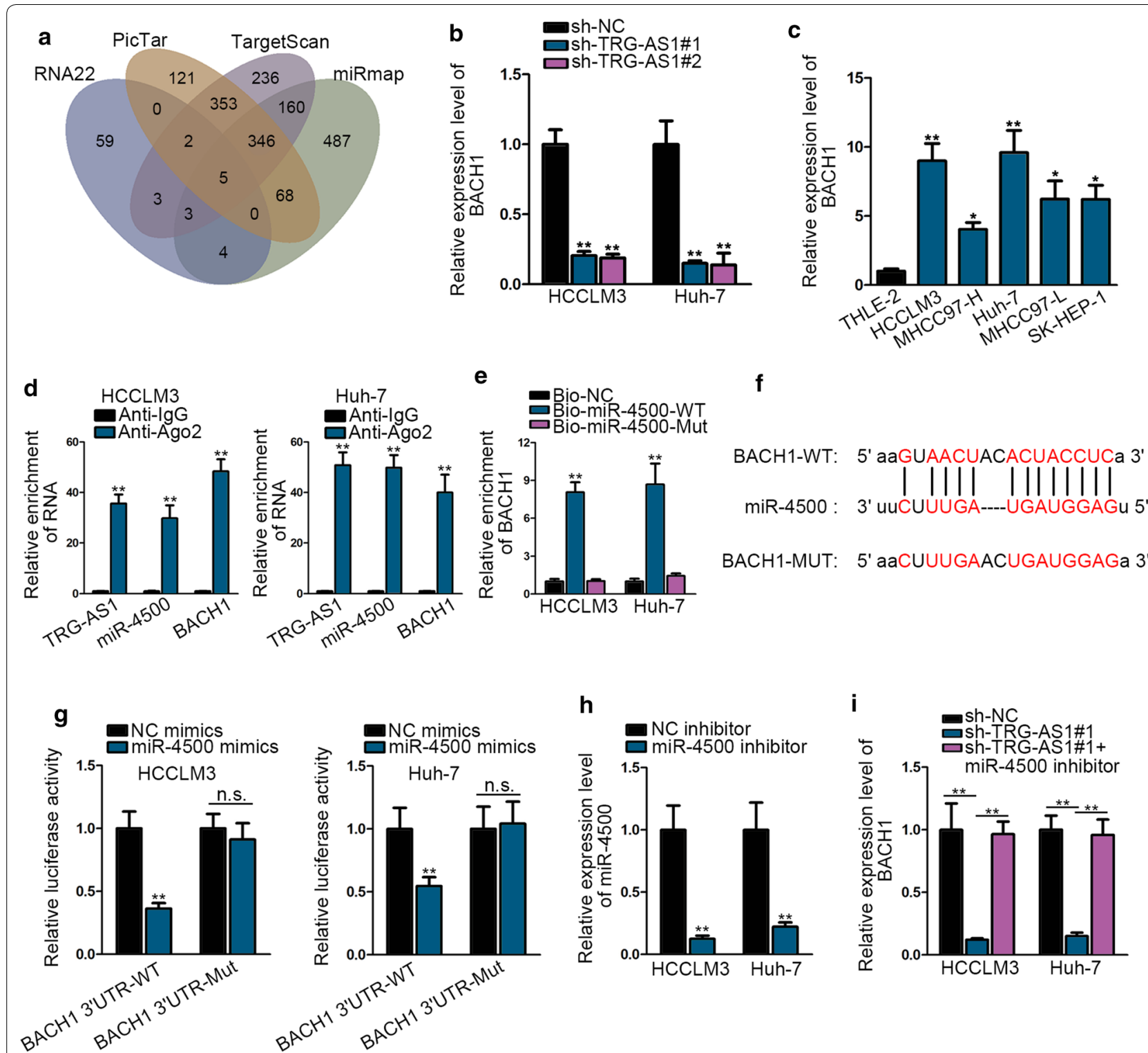

Fig. 4 BACH1 is modulated by the TRG-AS1 and miR-4500. a Underlying targets of miR-4500 were obtained by RNA22, PicTar, TargetScan and miRmap databases. b BACH1 expression was assessed using RT-qPCR in HCC cells transfected with sh-TRG-AS1. c BACH1 expression in HCC cell lines and THLE-2 was examined with RT-qPCR. $\mathbf{d}$ RIP assay was performed using Ago2 antibody and the enrichment of TRG-AS1, miR-4500 and BACH1 in both HCC cells was analyzed. e RNA pull down assay and RT-qPCR detected the enrichment of BACH1 with the function of Bio-miR-4500-WT/Mut. $\mathbf{f}$ The binding sites were predicted between BACH1 and miR-4500 via ENCORI database. $\mathbf{g}$ Luciferase reporter assay was used to verify combination of $\mathrm{BACH} 1$ and miR-4500. $\mathbf{h}$ Effects of miR-4500 inhibitor on miR-4500 expression in HCC cells were detected by RT-qPCR. i RT-qPCR analysis was used to determine the regulatory relationship among TRG-AS1, miR-4500 and $\mathrm{BACH1}$. ${ }^{\mathrm{P}}<0.05,{ }^{* *} \mathrm{P}<0.01$

TRG-AS1 silencing (Fig. 4b). We therefore detected the expression of BACH1 in HCC cells and FHC cell using RT-qPCR depending on these findings. As shown in Fig. 4c, BACH1 expression was noticeably up-regulated in HCC cells. RIP analysis showed that TRG-AS1/miR4500/BACH1 were enriched in Anti-Ago2 complex rather than Anti-IgG complex, indicating that they were co-existed in RNA-induced silencing complex (RISC)
(Fig. 4d). The results of RNA pull down suggested that BACH1 was remarkably elevated by Bio-miR-4500-WT, not by Bio- miR-4500-Mut in HCC cells (Fig. 4h). ENCORI predicted the complementary sites of BACH1 for miR-4500 (Fig. 4f). Furthermore, luciferase reporter assay uncovered that upregulation of miR-4500 dramatically cut down the luciferase activity of BACH1 3'UTRWT, not affected that of BACH1 $3^{\prime}$ UTR-Mut compared 
with NC mimics in HCC cells (Fig. 4g). Additionally, RTqPCR confirmed that miR-4500 expression was sharply silenced in HCCLM3 and Huh-7 cells treated with miR4500 inhibitor (Fig. 4h). As exhibited in Fig. 4i, BACH1 expression was remarkably suppressed with the knockdown of TRG-AS1, and was fully recovered due to miR4500 silence. Taken together, BACH1 is modulated by the TRG-AS1 and miR-4500.

\section{BACH1 acts as a tumor-promoting gene in $\mathrm{HCC}$}

We further explored the role of $\mathrm{BACH} 1$ in $\mathrm{HCC}$ via performing loss-of function assays. BACH1 expression was markedly silenced in HCCLM3 and Huh-7 cells treated with sh-BACH1\#1/\#2 (Fig. 5a). Next, BACH1 silencing resulted in the reduction of HCC cell proliferation (Fig. 5b, c). Likewise, the invasion and migration capacities of $\mathrm{HCC}$ cells were hampered by BACH1 down-regulation (Fig. 5d). Collectively, BACH1 contributes to HCC progression.

\section{TRG-AS1 contributes the progression of HCC by targeting} the miR-4500/BACH1 axis

To explore the interplay among TRG-AS1, miR-4500 and BACH1, HCCLM3 and Huh-7 cells were transfected with pcDNA3.1/BACH1 for rescue assays. As indicated in Fig. 6a, BACH1 was considerably up-regulated with the transfection of pcDNA3.1/BACH1. In colony formation assay, the colony formation ability of HCC cells was

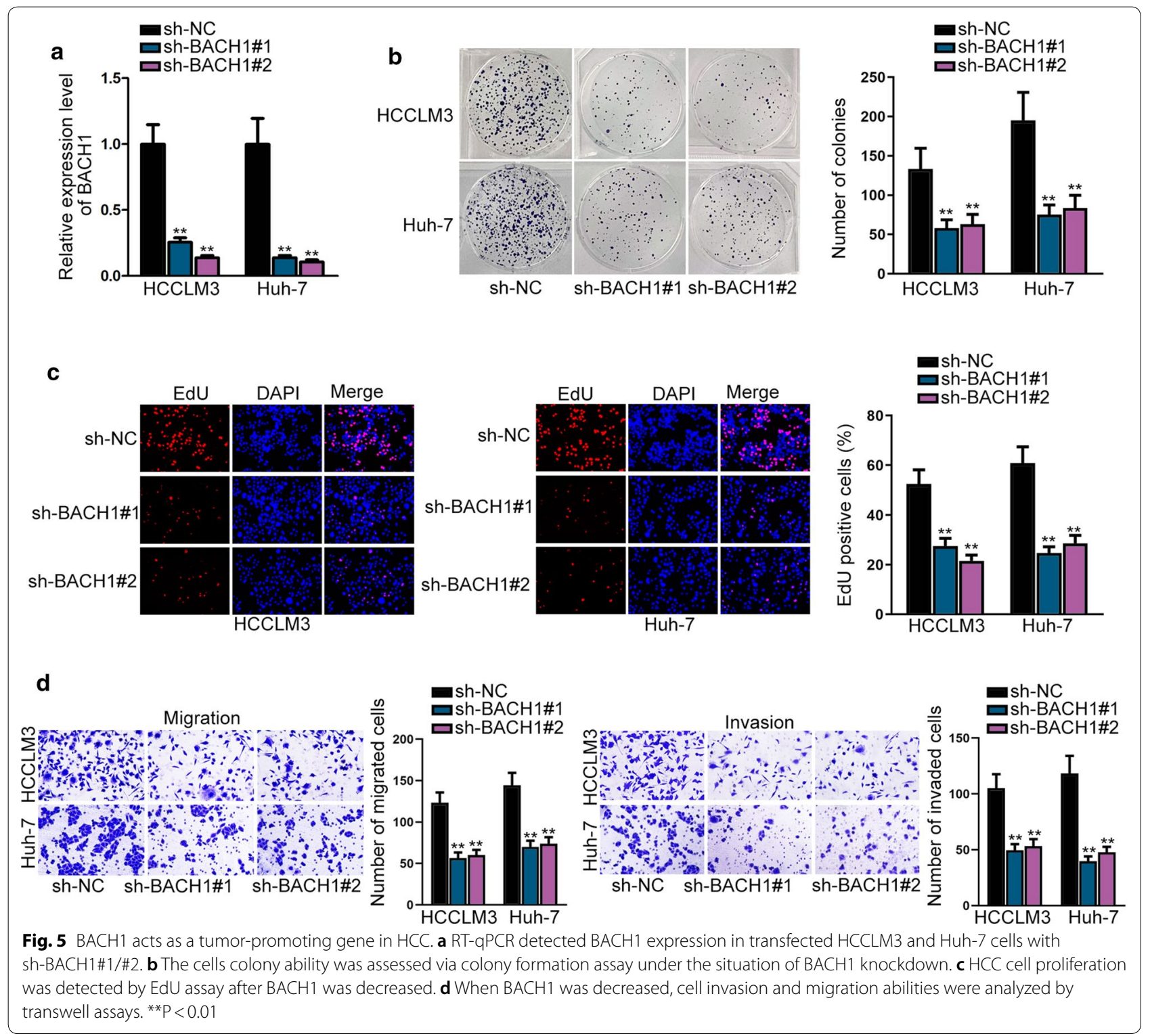




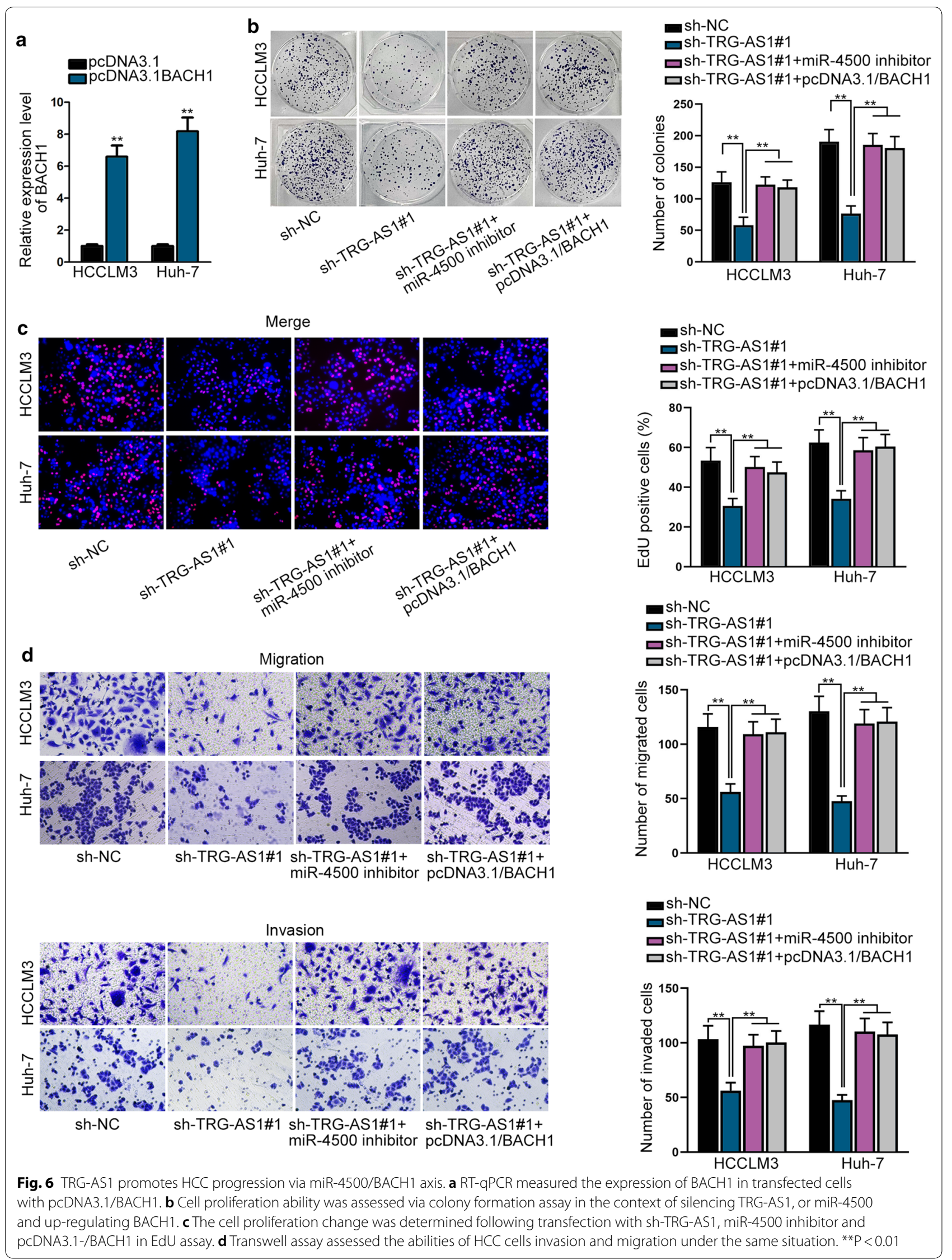


effectively inhibited due to TRG-AS1 knockdown, and then was fully rescued by miR-4500 down-regulation or BACH1 overexpression (Fig. 6b). Meanwhile, suppression of cell proliferation upon sh-TRG-AS1\#1 could be reversed by co-transfected with miR-4500 inhibitor or pcDNA3.1/BACH1 (Fig. 6c). The reduced invasion and migration abilities caused by TRG-AS1 down-regulation could also be wholly reversed by miR-4500 silence or BACH1 increase (Fig. 6D). Furthermore, the EMT process reversed by TRG-AS1 silencing was recovered by the inhibition of miR-4500 or the overexpression of BACH1 (Additional file 5: Figure S5A-B). Overall, TRG-AS1 promotes $\mathrm{HCC}$ progression via the miR-4500/BACH1 axis.

\section{Discussion}

Hepatocellular carcinoma $(\mathrm{HCC})$ is one of the most highly lethal cancers. At present, authoritative researches have revealed roles of lncRNAs as promoter or inhibitor of cancer-crucial genes in $\mathrm{HCC}$, which have capacity to regulate the biological behaviors of HCC cells $[16,17]$. In this study, we also tried to explore the molecular mechanism underneath HCC progression.

As for TRG-AS1, it recently has been reported to regulate glioblastoma progression [15]. Given that unknown role of TRG-AS1 in HCC, thus we mainly explored the role and function of TRG-AS1 in HCC in the present study. Here, we observed TRG-AS1 presented high expression in HCC cells. In the case of TRG-AS1 knockdown, HCC cell proliferation was effectively depressed.

Epithelial-mesenchymal transition (EMT) is key and pivotal marker for the tumor development or metastasis [18-20]. In the current study, we also detected the effect of TRG-AS1 silencing on EMT process in HCC. As a result, we found that the TRG-AS1 down-regulation effectively inhibited EMT progress. All in all, the finding demonstrated TRG-AS1 exerted tumor-promoting role in HCC, which was a possible target for HCC treatment.

LncRNAs can exert functions by binding with their downstream miRNAs to further perform its regulatory effect on target mRNAs. In this study, miR-4500 was the downstream miRNA of TRG-AS1, which could interact with TRG-AS1. Gain-of-functional assays, miR-4500 overexpression inhibited HCC cell proliferation migration, invasion and EMT progress. It has been reported that miR-4500 is abnormally expressed in other human cancers. For instance, miR-4500 has tumor-suppressive function in colorectal cancer and glioma and non-small cell lung cancer [21-23]. Furthermore, this study suggested that miR-4500 targeted $\mathrm{BACH} 1$. $\mathrm{BACH} 1$, as a member of cap ' $n$ ' collar (CNC) and basic region leucine zipper factor family, has been reported to participate in cancer progression [24]. For instance, BACH1 plays oncogene to accelerate CRC progression and is to be a useful prognostic factor for survival and metastasis [25]. Shajari et al. show that BACH1 is a key inducer in metastasis of prostate cancer [26]. In this study, BACH1 was overly expressed in $\mathrm{HCC}$ cells and its down-regulation remarkably restrained HCC progression by antiproliferation, anti-invasion/migration and anti-EMT progress. Finally, in the rescue assays, BACH1 expression was remarkably suppressed with the knockdown of TRGAS1, and was fully recovered due to miR-4500 silencing, indicating that TRG-AS1 acted as a competitive endogenous RNA (ceRNA) by adsorbing miR-4500 to regulate BACH1. Furthermore, TRG-AS1 was able to promote $\mathrm{HCC}$ progression via the miR-4500/BACH1 axis.

\section{Conclusion}

In summary, this study reported that lncRNA TRG-AS1 down-regulation inhibited proliferation, migration and invasion as well as EMT progress. TRG-AS1 may serve as a potential therapeutic target for HCC treatment.

\section{Supplementary information}

Supplementary information accompanies this paper at https://doi. org/10.1186/s12935-020-01440-3.

Additional file 1: Figure S1. A. RT-qPCR analysis of E-cadherin and $\mathrm{N}$-cadherin expression in two HCC cells transfected with sh-NC, sh-TRGAS1\#1, sh-TRG-AS1\#2. B. Western blot analysis was utilized to examine the protein levels of E-cadherin and N-cadherin in HCC cells transfected with sh-NC, sh-TRG-AS1\#1, sh-TRG-AS1\#2. **P $<0.01$.

Additional file 2: Figure S2. A. RNA pull down assay was performed using TRG-AS1 biotin probe and enrichment of mRNAs in both HCC cells was analyzed by RT-qPCR. ${ }^{* * P}<0.01$.

Additional file 3: Figure S3. A-B. The mRNA and protein levels of E-cadherin and N-cadherin were detected by RT-qPCR and western blot analyses in cells with ectopic expression of miR-4500. ${ }^{* *} \mathrm{P}<0.01$.

Additional file 4: Figure S4. A. The levels of candidate mRNAs were examined by RT-qPCR in HCCLM3 and Huh-7 cells transfected with miR4500 mimics. ${ }^{*} \mathrm{P}<0.01$.

Additional file 5: Figure S5. A-B. The mRNA and protein levels of EMTmakers (E-cadherin and N-cadherin) were measured in indicated HCC cells by RT-qPCR and western blot analyses, respectively.

\begin{abstract}
Abbreviations
IncRNAs: long non-coding RNAs; mRNA: messenger RNA; ceRNAs: competing endogenous RNAs; miRNA: microRNA; HCC: hepatocellular carcinoma; TRG-AS1:T cell receptor gamma locus antisense RNA 1; BACH1: BTB domain and CNC homolog 1; EMT: epithelial-mesenchymal transitions; Wt: wild-type; Mut: mutant; RT-qPCR: Quantitative real-time polymerase chain reaction; EdU: 5-ethynyl-20-deoxyuridine; DAPI: 4',6-diamidino-2-phenylindole; FISH: fluorescent in situ hybridization; RIP: RNA immunoprecipitation; IF: imunofluorescence staining; RISC: RNA-induced silencing complex; SD: standard deviation.
\end{abstract}

Acknowledgements

We appreciate the supports of our experimenters.

\section{Authors' contributions}

In this research, XS contributed to design and data curation. XW and RC performed experiments. JZ and WC analyzed the data and recorded the results. MF was responsible for investigation. XS and YQ wrote and revised the manuscript. All authors read and approved the final manuscript. 


\section{Funding}

This work was supported by Study on the Integrin/FAK signaling mechanism of Talin 1 affecting the invasion and metastasis of lost-nest apoptosis in HCC cells.

\section{Availability of data and materials}

Not applicable.

\section{Ethics approval and consent to participate}

Not applicable.

\section{Consent for publication}

Authors confirmed that this work can be published. The content of this manuscript is original and it has not yet been accepted or published elsewhere.

\section{Competing interests}

The authors declare that they have no competing interests.

\section{Author details}

${ }^{1}$ Department of Emergency Surgery, the First Affiliated Hospital of Anhui Medical University, Hefei 230000, Anhui, China. ${ }^{2}$ Department of Hepatobiliary Surgery, the First Affiliated Hospital of Anhui Medical University, No.218 Jixi Road, Hefei 230000, Anhui, China. ${ }^{3}$ Department of Emergency, the First Affiliated Hospital of University of Science and Technology of China, Hefei 230001, Anhui, China.

Received: 7 May 2020 Accepted: 20 July 2020

Published online: 04 August 2020

\section{References}

1. Chacko S, Samanta S. Hepatocellular carcinoma: a life-threatening disease. Biomed Pharmacother. 2016;84:1679-88.

2. Bellissimo F, Pinzone MR, Cacopardo B, Nunnari G. Diagnostic and therapeutic management of hepatocellular carcinoma. World J Gastroenterol. 2015;21(42):12003-21.

3. Hammad A, Kaido T, Ogawa K, Fujimoto Y, Uemura T, Mori A, Hatano E, Okajima H, Uemoto S. Liver transplantation for advanced hepatocellular carcinoma in patients with Child-Pugh A and B. Surg Today. 2016;46(2):248-54.

4. Yeung RH, Chapman TR, Bowen SR, Apisarnthanarax S. Proton beam therapy for hepatocellular carcinoma. Expert Rev Anticancer Ther. 2017;17(10):911-24.

5. Chen S, Wang H, Zhang W, Chen J, Lu P. Clinical application of laparoscopy-assisted and percutaneous radiofrequency ablation for hepatocellular carcinoma. J Southern Med Univ. 2018;38(9):1147-50.

6. Anastasiadou E, Jacob LS, Slack FJ. Non-coding RNA networks in cancer. Nat Rev Cancer. 2018;18(1):5-18.

7. Yang Y, Chen L, Gu J, Zhang H, Yuan J, Lian Q, Lv G, Wang S, Wu Y, Yang YT, et al. Recurrently deregulated IncRNAs in hepatocellular carcinoma. Nat Commun. 2017:8:14421.

8. Bhan A, Soleimani M, Mandal SS. Long noncoding RNA and cancer: a new paradigm. Cancer Res. 2017;77(15):3965-81.

9. Yang J, Li C, Mudd A, Gu X. LncRNA PVT1 predicts prognosis and regulates tumor growth in prostate cancer. Biosci Biotechnol Biochem. 2017:81(12):2301-6.
10. Xiao J, Lv Y, Jin F, Liu Y, Ma Y, Xiong Y, Liu L, Zhang S, Sun Y, Tipoe GL, et al. LnCRNA HANR promotes tumorigenesis and increase of chemoresistance in hepatocellular carcinoma. Cell Physiol Biochem. 2017;43(5):1926-38.

11. Huang $Y$, Xiang B, Liu Y, Wang Y, Kan H. LncRNA CDKN2B-AS1 promotes tumor growth and metastasis of human hepatocellular carcinoma by targeting let-7c-5p/NAP1L1 axis. Cancer Lett. 2018;437:56-66.

12. Sheng N, Li Y, Qian R, Li Y. The clinical significance and biological function of IncRNA RGMB-AS1 in hepatocellular carcinoma. Biomed Pharmacother. 2018;98:577-84.

13. Xu JH, Chang WH, Fu HW, Yuan T, Chen P. The mRNA, miRNA and IncRNA networks in hepatocellular carcinoma: an integrative transcriptomic analysis from Gene Expression Omnibus. Mol Med Rep. 2018;17(5):6472-82.

14. Wang Y, Yang L, Chen T, Liu X, Guo Y, Zhu Q, Tong X, Yang W, Xu Q, Huang $D$, et al. A novel IncRNA MCM3AP-AS1 promotes the growth of hepatocellular carcinoma by targeting miR-194-5p/FOXA1 axis. Mol Cancer. 2019;18(1):28.

15. Xie H, Shi S, Chen Q, Chen Z. LncRNA TRG-AS1 promotes glioblastoma cell proliferation by competitively binding with miR-877-5p to regulate SUZ12 expression. Pathol Res Pract. 2019;215(8):152476.

16. Qiu L, Tang Q, Li G, Chen K. Long non-coding RNAs as biomarkers and therapeutic targets: recent insights into hepatocellular carcinoma. Life Sci. 2017;191:273-82.

17. He Y, Meng XM, Huang C, Wu BM, Zhang L, Lv XW, Li J. Long noncoding RNAs: novel insights into hepatocelluar carcinoma. Cancer Lett. 2014;344(1):20-7.

18. Lamouille $S, X u J$, Derynck R. Molecular mechanisms of epithelial-mesenchymal transition. Nat Rev Mol Cell Biol. 2014;15(3):178-96.

19. Mittal V. Epithelial mesenchymal transition in tumor metastasis. Ann Rev Pathol. 2018;13:395-412.

20. Zhang Y, Weinberg RA. Epithelial-to-mesenchymal transition in cancer: complexity and opportunities. Front Med. 2018;12(4):361-73.

21. Li ZW, Xue M, Zhu BX, Yue CL, Chen M, Qin HH. microRNA-4500 inhibits human glioma cell progression by targeting IGF2BP1. Biochem Biophys Res Commun. 2019;513(4):800-6.

22. Yu FY, Tu Y, Deng Y, Guo C, Ning J, Zhu Y, Lv X, Ye H. MiR-4500 is epigenetically downregulated in colorectal cancer and functions as a novel tumor suppressor by regulating HMGA2. Cancer Biol Ther. 2016;17(11):1149-57.

23. Zhang L, Qian J, Qiang Y, Huang H, Wang C, Li D, Xu B. Down-regulation of miR-4500 promoted non-small cell lung cancer growth. Cell Physiol Biochem. 2014;34(4):1166-74.

24. Davudian S, Mansoori B, Shajari N, Mohammadi A, Baradaran B. BACH1, the master regulator gene: a novel candidate target for cancer therapy. Gene. 2016;588(1):30-7.

25. Zhu GD, Liu F, OuYang S, Zhou R, Jiang FN, Zhang B, Liao WJ. BACH1 promotes the progression of human colorectal cancer through $\mathrm{BACH} 1 /$ CXCR4 pathway. Biochem Biophys Res Commun. 2018;499(2):120-7.

26. Shajari N, Davudian S, Kazemi T, Mansoori B, Salehi S, Khaze Shahgoli $\checkmark$, Shanehbandi D, Mohammadi A, Duijf PHG, Baradaran B. Silencing of $\mathrm{BACH} 1$ inhibits invasion and migration of prostate cancer cells by altering metastasis-related gene expression. Artif Cells Nanomed Biotechnol. 2018;46(7):1495-504.

\section{Publisher's Note}

Springer Nature remains neutral with regard to jurisdictional claims in published maps and institutional affiliations. 